\title{
Rodents rake in rewards
}

In new research aimed at identifying brain changes that underlie the development of tool-use ability, scientists have taught captive rodents to use tools. This is the first report of such training in rodents.

Atsushi Iriki and colleagues at the Brain Science Institute (RIKEN, Saitama, Japan) trained a group of five degus (Octodon degus), small rodents native to Chile, to reach through a fence, grasp a rake and use it to retrieve a food reward that would be otherwise out of reach. They used a stepby-step training procedure with increasing complexity. At the first level, the reward was placed immediately in front of the rake, so that the animal only had to pull the rake toward itself. The distance between the rake and the reward was increased in the second level of training. In the third level of training, the reward was placed to the side of the rake, requiring the animal to move the rake laterally before pulling it in order to retrieve the reward. In the final level, the reward was

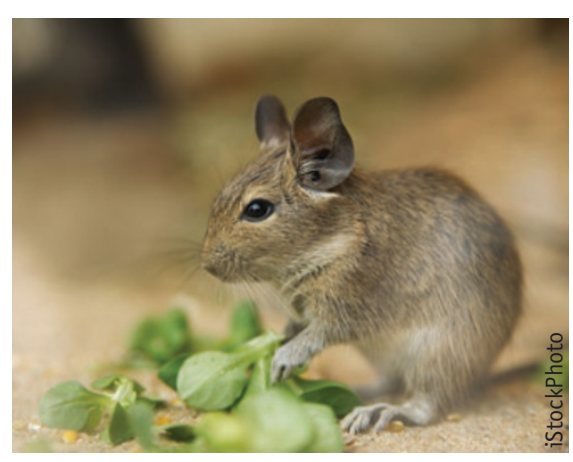

Iriki's group had previously used a similar procedure to train Japanese macaques, which do not tend to use tools in the wild. As the macaques learned to use the rake to retrieve food rewards, the researchers observed concomitant changes in gene expression and in circuitry in the intraparietal cortex, an area of the brain involved in cognition and processing of sensory input. Iriki believes it is reasonable to assume that similar changes may take place in the rodent brain as a result of tool-use training, and his group has begun studies to address this question. The group is also investigating potential brain changes in marmosets.

Iriki's work may offer insight into the development of tool use in early humans. Tool use is commonly considered to be a mark of advanced intelligence. But the new findings suggest instead that the brain structures involved in tool use may be present, albeit dormant, in many animals. Monica Harrington

\section{SWEET TASTE OF NOTHING}

As those who have tried to lose weight might attest, artificially sweetened drinks and desserts never quite compare with the real thing. A new study shows that our preference for sugary sweets might not just be rooted in their taste-we may derive pleasure from calorie content alone.

Previous studies have shown that tasty foods stimulate the brain's dopamine reward system, which can override animals' homeostasis mechanisms and lead to overeating and obesity. A new study led by Ivan de Araujo at Duke University (Durham, NC) explored dopamine activity in mice that drank 'delicious' sugar water-but could not taste it (Neuron 57, 930-941; 2008).

The researchers used genetically modified mice that lacked TRPM5, a protein necessary for perceiving sweet taste. They gave mice a choice between regular drinking water and water sweetened with sucrose. Like wild-type mice, knockout mice showed a distinct preference for sugar water, despite being 'blind' to its taste. When the scientists gave another group of mice a choice between plain water and water sweetened with noncaloric sucralose, knockout mice showed no preference, whereas wildtype mice preferred the sweetened water.

The researchers then examined dopamine concentrations using microdialysis probes that were implanted in the mice. As expected, dopamine concentrations in wild-type mice increased when they consumed either sucrose or sucralose. In knockout mice, dopamine concentrations increased after sucrose consumption, but not when mice consumed artificial sweetener. This suggests that after ingestion, the metabolic effects of sucrose stimulate brain reward circuits.

The 'double enjoyment' of sweets may be nature's way of ensuring the most efficient identification of calorie-rich foods. Though sweet taste helps animals spot these foods quickly, de Araujo tells Lab Animal, the "real reward" they're after is calorie content. "Thus, we need a taste-independent system that will take into account the metabolic consequence of ingesting a particular compound." If a sweet food turns out to lack calories, the attraction to that food might eventually be overridden. Previous studies have shown that rats that are given saccharine over time become less responsive to sweet taste and are more likely to compensate with calories from other sources.

It is still early to predict how these findings might apply to humans. "In humans food intake is a fairly complex process that involves cognitive and social cues," says de Araujo. The study implies, however, that attempts to curb our eating by replacing rich foods with low-calorie imitations "might not be efficient in the long run." Karen Marron 